CREAT. MATH. INFORM.

Volume 29 (2020), No. 2,

Pages 191 - 203

\title{
Denumerably many positive solutions for fractional order boundary value problems
}

\author{
K. RAJENDRA Prasad, MAHAmmad KHUdDUSH and M. RASHMita
}

\begin{abstract}
The sufficient conditions are derived for the existence of denumerably many positive solutions for fractional order boundary value problem

$$
\begin{gathered}
\mathfrak{D}_{0^{+}}^{\varepsilon}\left[\phi\left[\mathfrak{D}_{0^{+}}^{\eta} \omega(t)\right]\right]+f(\omega(t))=0,0<t<1, \\
\omega(0)=\omega^{\prime}(0)=\mathfrak{D}_{0^{+}}^{\eta} \omega(0)=0, \\
\mathfrak{D}_{0^{+}}^{\varepsilon} \omega(1)=I_{0^{+}}^{\delta} \omega(1)
\end{gathered}
$$

where $\mathfrak{D}_{0^{+}}^{\varepsilon}, \mathfrak{D}_{0^{+}}^{\eta}$ denote fractional derivatives of Riemann-Liouville type with $0<\varepsilon<1,2<\eta \leq 3, I_{0^{+}}^{\delta}$ $(\delta \in \mathbb{R})$ denotes Riemann-Liouville fractional integral, $\phi: \mathbb{R} \rightarrow \mathbb{R}$ is an increasing homeomorphism and positive homomorphism operator (IHPHO), by applying Hölder's inequality and Krasnoselskii's cone fixed point theorem in a Banach space.
\end{abstract}

\section{INTRODUCTION}

The fractional derivatives are used for a better description of considered material properties, mathematical modelling based on enhanced rheological models naturally leads to differential equations of fractional order and to the necessity of the formulation of initial conditions to such equations. Applied problems require definitions of fractional derivatives allowing the utilization of physically interpretable initial conditions. It should be noted that most papers and books on fractional calculus are devoted to the solvability of linear initial fractional differential equations in terms of special functions. Recently, the researchers are shown a great deal of interest in establishing existence and multiplicity of solutions (or positive solutions) of nonlinear initial fractional differential equations by the use of techniques of nonlinear analysis, see [7, 12,36] and reference therein. In fact, boundary conditions have the same requirements. However, few papers have considered the boundary value problems of fractional differential equations, see $[25,15,16]$.

In [30], Y. Tian and Z. Bai considered the $p$-Laplacian fractional order boundary value problem

$$
\begin{gathered}
\mathfrak{D}_{0^{+}}^{\beta}\left(\phi_{p}\left(\mathfrak{D}_{0^{+}}^{\alpha} \omega(t)\right)\right)=f(t, \omega(t)), t \in(0,1) \\
\omega(0)=\omega^{\prime}(0)=0, \omega^{\prime}(1)=a \omega^{\prime}(\xi) \\
\mathfrak{D}_{0^{+}}^{\alpha} \omega(0)=0, \mathfrak{D}_{0^{+}}^{\alpha} \boldsymbol{\omega}(1)=b \mathfrak{D}_{0^{+}}^{\alpha} \boldsymbol{\omega}(\eta),
\end{gathered}
$$

and established existence of positive solutions by applying monotone iterative method and the fixed point index theory on cones. In [9], Ege and Topal considered the fractional

Received: 25.02.2020. In revised form: 22.08.2020. Accepted: 29.08.2020

2010 Mathematics Subject Classification. 47B34, 34A08, 34B18.

Key words and phrases. Fractional derivative, homeomorphism, homomorphism, cone, Krasnoselskii's fixed point theorem, positive solutions.

Corresponding author: Mahammad Khuddush; khuddush89@gmail.com 
boundary value problem with $\mathrm{IHPHO}$,

$$
\begin{gathered}
\mathcal{C}_{\mathfrak{D}^{q}}\left(\phi\left({ }^{\mathcal{C}} \mathfrak{D}^{r} \omega(t)\right)\right)+f(t, \omega(t))=0, \\
\alpha_{1} \omega(0)-\beta_{1} \omega^{\prime}(0)=-\gamma_{1} \omega\left(\xi_{1}\right), \alpha_{2} x(1)+\beta_{2} \omega^{\prime}(1)=-\gamma_{2} \omega\left(\xi_{2}\right) \\
\mathcal{C}_{\mathfrak{D}^{r} \omega(0)=0,0<q \leq 1<r \leq 2,0<t<1}
\end{gathered}
$$

and established existence of positive solutions by utilizing Krasnoselskii's and LeggetWilliams cone fixed point.

Inspired by the works mentioned above, in this paper we consider Riemann-Liouville fractional order boundary value problem with IHPHO,

$$
\left\{\begin{array}{c}
\mathfrak{D}_{0^{+}}^{\varepsilon}\left[\phi\left[\mathfrak{D}_{0^{+}}^{\eta} \omega(t)\right]\right]+f(\omega(t))=0,0<t<1, \\
\omega(0)=\omega^{\prime}(0)=\mathfrak{D}_{0^{+}}^{\eta} \omega(0)=0, \\
\mathfrak{D}_{0^{+}}^{\varepsilon} \omega(1)=I_{0^{+}}^{\delta} \omega(1)
\end{array}\right.
$$

where $\mathfrak{D}_{0^{+}}^{\varepsilon}, \mathfrak{D}_{0^{+}}^{\eta}$ denote fractional derivatives of Riemann-Liouville type with $0<\varepsilon<1$, $2<\eta \leq 3, I_{0^{+}}^{\delta}\left(\delta \in \mathbb{R}^{+}\right)$denotes Riemann-Liouville fractional integral and establish the existence of denumerably many positive solutions.

We assume that the following conditions are satisfied throughout the paper:

$\left(H_{1}\right) f:[0,+\infty) \rightarrow[0,+\infty)$ is continuous,

$\left(H_{2}\right)$ For $0 \leq \tau \leq 1,0<\psi(\tau)<\infty$, where

$$
\psi(\tau)=\phi^{-1}\left(\frac{\tau^{\varepsilon}}{\Gamma(\varepsilon+1)}\right), 0<\varepsilon \leq 1 .
$$

\section{KERNEL AND IT'S BOUNDS}

In this section, the basic definitions and lemmas which are useful for our later discussions are stated and constructed kernel for the boundary value problem (1.1) later we estimated bounds for the kernel.

Definition 2.1. [14] The Riemann-Liouville(RL) fractional integral of order $\delta$ of a function $\mathfrak{T}:(0, \infty) \rightarrow \mathbb{R}$ is defined by

$$
\mathcal{I}_{0^{+}}^{\delta} \mathfrak{T}(s)=\int_{0}^{s} \frac{(s-x)^{\delta-1}}{\Gamma(\delta)} \mathfrak{T}(x) d x, \delta>0 .
$$

Lemma 2.1. [14, 26] The general solution to $\mathfrak{D}_{0^{+}}^{\gamma} \mathfrak{T}(t)=0$ with $\gamma \in(m-1, m]$ and $m>1$ is the function

$$
\mathfrak{T}(t)=\sum_{\ell=1}^{m} d_{\ell} t^{\gamma-\ell},
$$

where $d_{\ell}$ is a real number.

Lemma 2.2. $[14,26]$ Let $\gamma>0$. Then for any function $\mathfrak{T}:(0, \infty) \rightarrow \mathbb{R}$, we have

$$
\mathfrak{D}_{0^{+}}^{-\gamma} \mathfrak{D}_{0^{+}}^{\gamma} \mathfrak{T}(t)=\mathfrak{T}(t)+\sum_{\ell=1}^{m} d_{\ell} t^{\gamma-\ell},
$$

where $d_{\ell}$ is a real number and $m \in \mathbb{Z}$ is the smallest integer greater that or equal to $\gamma$.

Lemma 2.3. Suppose $\left(H_{1}\right)$ and $\left(H_{2}\right)$ hold. Let $\eta \in(1,2]$ and $g \in C^{2}[0,1]$. The boundary value problem

$$
\begin{gathered}
\mathfrak{D}_{0^{+}}^{\eta} \omega(t)+g(t)=0, t \in(0,1), \\
\omega(0)=\omega^{\prime}(0)=0, \mathfrak{D}_{0^{+}}^{\varepsilon} \omega(1)=I_{0^{+}}^{\delta} \omega(1),
\end{gathered}
$$


has a unique solution,

$$
\omega(t)=\int_{0}^{1} \chi(t, \tau) g(\tau) d \tau+\int_{0}^{1} \mathcal{H}(t, \tau) \int_{0}^{1} \chi(\tau, s) g(s) d s d \tau
$$

where

$$
\begin{aligned}
& \chi(t, \tau)=\frac{1}{\Gamma(\eta)}\left\{\begin{array}{lr}
t^{\eta-1}(1-\tau)^{\eta-\varepsilon-1}-(t-\tau)^{\eta-1}, & \tau \leq t, \\
t^{\eta-1}(1-\tau)^{\eta-\varepsilon-1}, & t \leq \tau,
\end{array}\right. \\
& \mathcal{H}(t, \tau)=\frac{\Gamma(\eta-\varepsilon) \Gamma(\eta+\delta)}{[\Gamma(\eta+\delta)-\Gamma(\eta-\varepsilon)] \Gamma(\eta) \Gamma(\delta)} t^{\eta-1}(1-\tau)^{\delta-1} .
\end{aligned}
$$

Proof. The equivalent fractional integral equation to the equation (2.2) is given by

$$
\omega(t)=c_{1} t^{\eta-1}+c_{2} t^{\eta-2}+c_{3} t^{\eta-3}-\int_{0}^{t} \frac{(t-\tau)^{\eta-1}}{\Gamma(\eta)} g(\tau) d \tau,
$$

where $c_{1}, c_{2}$ and $c_{3}$ are constants. Applying boundary conditions 2.3 into the above equation, one can obtain $c_{2}=c_{3}=0$ and

$$
c_{1}=\int_{0}^{1} \frac{(1-\tau)^{\eta-\varepsilon-1}}{\Gamma(\eta)} g(\tau) d \tau+\frac{\Gamma(\eta-\varepsilon)}{\Gamma(\eta)} I_{0^{+}}^{\delta} \omega(1) .
$$

Therefore,

$$
\omega(t)=\int_{0}^{1} \chi(t, \tau) g(\tau) d \tau+\frac{\Gamma(\eta-\varepsilon)}{\Gamma(\eta)} t^{\eta-1} I_{0^{+}}^{\delta} \omega(1) .
$$

By simple calculations, we get

$$
\begin{aligned}
& I_{0^{+}}^{\delta} \omega(1)=\frac{\Gamma(\eta+\delta)}{\Gamma(\eta+\delta)-\Gamma(\eta-\varepsilon)} {\left[\int_{0}^{1} \frac{(1-\tau)^{\delta-1}}{\Gamma(\delta)}\right.} \\
&\left.\times \int_{0}^{1} \chi(\tau, s) g(s) d s d \tau\right] .
\end{aligned}
$$

Hence,

$$
\omega(t)=\int_{0}^{1} \chi(t, \tau) g(\tau) d \tau+\int_{0}^{1} \mathcal{H}(t, \tau) \int_{0}^{1} \chi(\tau, s) g(s) d s d \tau
$$

Lemma 2.4. The kernel $\chi(t, \tau)$ have the following properties:

(i) $\chi(t, \tau) \geq 0$ and continuous on $[0,1] \times[0,1]$,

(ii) $\chi(t, \tau) \leq \chi(1, \tau)$ for $t, \tau \in[0,1]$,

(iii) there exists $\varrho \in\left(0, \frac{1}{2}\right)$ such that $\varrho^{\eta-1} \chi(1, \tau) \leq \chi(t, \tau)$ for $t \in[\varrho, 1-\varrho], \tau \in[0,1]$.

Proof. By simple calculations, (i) and (ii) can be established easily. We prove (iii). For $0 \leq \tau \leq t \leq 1$, let

$$
F(t, \tau)=\frac{t^{\eta-1}(1-\tau)^{\eta-\varepsilon-1}}{\Gamma(\eta)}-\frac{(t-\tau)^{\eta-1}}{\Gamma(\eta)} .
$$

Then for $\varrho \in\left(0, \frac{1}{2}\right)$, we have

$$
\begin{aligned}
F(t, \tau) & \geq t^{\eta-1}\left[\frac{(1-\tau)^{\eta-\varepsilon-1}}{\Gamma(\eta)}-\frac{(1-\tau)^{\eta-1}}{\Gamma(\eta)}\right] \\
& \geq \varrho^{\eta-1}\left[\frac{(1-\tau)^{\eta-\varepsilon-1}}{\Gamma(\eta)}-\frac{(1-\tau)^{\eta-1}}{\Gamma(\eta)}\right] \\
& \geq \varrho^{\eta-1} \chi(1, \tau) .
\end{aligned}
$$


Lemma 2.5. Suppose $\Gamma(\eta+\delta)>\Gamma(\eta-\varepsilon)$ and $\varrho \in\left(0, \frac{1}{2}\right]$. Then

$$
\min _{t \in[\varrho, 1-\varrho]} \mathcal{H}(t, \tau) \geq \varrho^{\eta-1} \max _{t \in[0,1]} \mathcal{H}(t, \tau) .
$$

Proof. From Lemma 2.2,

$$
\frac{\min _{t \in[\varrho, 1-\varrho]} \mathcal{H}(t, \tau)}{\max _{t \in[0,1]} \mathcal{H}(t, \tau)}=\frac{\varrho^{\eta-1}(1-\tau)^{\delta-1}}{(1-\tau)^{\delta-1}}=\varrho^{\eta-1} .
$$

Lemma 2.6. Suppose $\left(H_{1}\right)$ and $\left(H_{2}\right)$ hold, then the unique solution of (1.1) is given by

$$
\begin{aligned}
\omega(t) & =\int_{0}^{1} \chi(t, \tau) \phi^{-1}\left(\mathcal{I}_{0^{+}}^{\varepsilon}(f(\omega(\tau)))\right) d \tau \\
& +\int_{0}^{1} \mathcal{H}(t, \tau) \int_{0}^{1} \chi(\tau, s) \phi^{-1}\left(\mathcal{I}_{0^{+}}^{\varepsilon}(f(\omega(s)))\right) d s d \tau .
\end{aligned}
$$

Proof. The problem (1.1) can be written as

$$
\begin{gathered}
\mathfrak{D}_{0^{+}}^{\varepsilon} v(t)+f(\omega(t))=0, \\
v(0)=0,
\end{gathered}
$$

by letting $\mathfrak{D}_{0^{+}}^{\eta} \omega(t)=u(t)$ and $v=\phi(u)$ with the condition $\mathfrak{D}_{0+}^{\eta} \omega(0)=0$. The solution of the problem is given by

By the condition $v(0)=0$, we get

$$
v(t)=c t^{\varepsilon-1}-\mathcal{I}_{0^{+}}^{\varepsilon}(f(\omega(t))) .
$$

$$
v(t)=-\mathcal{I}_{0^{+}}^{\varepsilon}(f(\omega(t)))
$$

Hence, from the Lemma 2.4, the problem

$$
\left\{\begin{array}{c}
\mathfrak{D}_{0^{+}}^{\eta} \omega(t)=-\phi^{-1}\left(I_{0^{+}}^{\varepsilon}(f(\omega(t)))\right) \\
\mathfrak{D}_{0^{+}}^{\varepsilon} \omega(1)=I_{0^{+}}^{a} \omega(1) \\
\omega(0)=\omega^{\prime}(0)=0
\end{array}\right.
$$

has a unique solution given by

$$
\begin{aligned}
\omega(t) & =\int_{0}^{1} \chi(t, \tau) \phi^{-1}\left(\mathcal{I}_{0^{+}}^{\varepsilon}(f(\omega(\tau)))\right) d \tau \\
& +\int_{0}^{1} \mathcal{H}(t, \tau) \int_{0}^{1} \chi(\tau, s) \phi^{-1}\left(\mathcal{I}_{0^{+}}^{\varepsilon}(f(\omega(s)))\right) d s d \tau
\end{aligned}
$$

We denote the Banach space $C^{4}([0,1], \mathbb{R})$ by $\mathcal{X}$ with the norm

$$
\|\omega\|=\max _{t \in[0,1]}|\omega(t)| \text {. }
$$

For $\varrho \in(0,1 / 2)$, the cone $\mathcal{P}_{\varrho} \subset \mathcal{X}$ is defined by

$$
\mathcal{P}_{\varrho}=\left\{\omega \in \mathcal{X}: \omega(t) \geq 0, \min _{t \in[\varrho, 1-\varrho]} \omega(t) \geq \varrho^{\eta-1}\|\omega(t)\|\right\},
$$

For any $\omega \in \mathcal{P}_{\varrho}$, define an operator $\mathcal{A}: \mathcal{P}_{\varrho} \rightarrow \mathcal{X}$ by

$$
\begin{aligned}
(\mathcal{A} \omega)(t) & =\int_{0}^{1} \chi(t, \tau) \phi^{-1}\left(\mathcal{I}_{0^{+}}^{\varepsilon}(f(\omega(\tau)))\right) d \tau \\
+ & \int_{0}^{1} \mathcal{H}(t, \tau) \int_{0}^{1} \chi(\tau, s) \phi^{-1}\left(\mathcal{I}_{0^{+}}^{\varepsilon}(f(\omega(s)))\right) d s d \tau
\end{aligned}
$$


Lemma 2.7. Assume that $\left(H_{1}\right)$ and $\left(H_{2}\right)$ hold. Then for each $\varrho \in(0,1 / 2), \mathcal{A}\left(\mathcal{P}_{\varrho}\right) \subset \mathcal{P}_{\varrho}$ and $\mathcal{A}: \mathcal{P}_{\varrho} \rightarrow \mathcal{P}_{\varrho}$ is completely continuous.

Proof. Let $\varrho \in(0,1 / 2)$. Since $f(\omega(\tau))$ is nonnegative for $\tau \in[0,1], \omega \in \mathcal{P}_{\varrho}$. Since $\chi(t, \tau)$ is nonnegative for all $t, \tau \in[0,1]$, it follows that $\mathcal{A}(\omega(t)) \geq 0$ for all $t \in$ $[0,1], \omega \in \mathcal{P}_{\varrho}$. Now, by Lemma 2.5 , we have

$$
\begin{aligned}
& \min _{t \in[\varrho, 1-\varrho]}(\mathcal{A} \omega)(t)= \min _{t \in[\varrho, 1-\varrho]}\left\{\int_{0}^{1} \chi(t, \tau) \phi^{-1}\left(\mathcal{I}_{0^{+}}^{\varepsilon}(f(\omega(\tau)))\right) d \tau\right. \\
&\left.\quad+\int_{0}^{1} \mathcal{H}(t, \tau) \int_{0}^{1} \chi(\tau, x) \phi^{-1}\left(\mathcal{I}_{0^{+}}^{\varepsilon}(f(\omega(x)))\right) d x d \tau\right\} \\
& \geq \int_{0}^{1} \chi(1, \tau) \phi^{-1}\left(\mathcal{I}_{0^{+}}^{\varrho}(f(\omega(\tau)))\right) d \tau \\
& \quad+\max _{t \in[0,1]} \int_{0}^{1} \mathcal{H}(t, \tau) \int_{0}^{1} \chi(\tau, x) \phi^{-1}\left(\mathcal{I}_{0^{+}}^{\varepsilon}(f(\omega(x)))\right) d x d \tau \\
& \geq \varrho^{\eta-1} \max _{t \in[0,1]}\left\{\int_{0}^{1} \chi(t, \tau) \phi^{-1}\left(\mathcal{I}_{0^{+}}^{\varepsilon}(f(\omega(\tau)))\right) d \tau\right.\left.\quad+\int_{0}^{1} \mathcal{H}(t, \tau) \int_{0}^{1} \chi(\tau, x) \phi^{-1}\left(\mathcal{I}_{0^{+}}^{\varepsilon}(f(\omega(x)))\right) d x d \tau\right\} \\
& \geq \varrho^{\eta-1} \max _{t \in[0,1]}|\mathcal{A} \omega(t)| .
\end{aligned}
$$

Thus $\mathcal{A}\left(\mathcal{P}_{\varrho}\right) \subset \mathcal{P}_{\varrho}$. Therefore, the operator $\mathcal{A}$ is completely continuous by standard methods and by the Arzela-Ascoli theorem.

\section{DENUMERABLY INFINITELY MANY POSITIVE SOLUTIONS}

In this section, we establish the existence of denumerably many positive solutions for bvp (1.1) by applying Krasnoselskii's fixed point theorem and Hölder's inequality. Firstly, we state fixed point theorem

Theorem 3.1 (Krasnoselskii's). [13] Let $\mathcal{E}$ be a cone in a Banach space $\mathcal{X}$ and $\Omega_{1}, \Omega_{2}$ are open sets with $0 \in \Omega_{1}, \bar{\Omega}_{1} \subset \Omega_{2}$. Let $\mathcal{A}: \mathcal{E} \cap\left(\bar{\Omega}_{2} \backslash \Omega_{1}\right) \rightarrow \mathcal{E}$ be a completely continuous operator such that

(a) $\|\mathcal{A} z\| \leq\|z\|, z \in \mathcal{E} \cap \partial \Omega_{1}$, and $\|\mathcal{A} z\| \geq\|z\|, z \in \mathcal{E} \cap \partial \Omega_{2}$, or

(b) $\|\mathcal{A} z\| \geq\|z\|, z \in \mathcal{E} \cap \partial \Omega_{1}$, and $\|\mathcal{A} z\| \leq\|z\|, z \in \mathcal{E} \cap \partial \Omega_{2}$.

Then $\mathcal{A}$ has a fixed point in $\mathcal{E} \cap\left(\bar{\Omega}_{2} \backslash \Omega_{1}\right)$.

Theorem 3.2. (Hölder's) Let $h \in L^{p}[0,1]$ and $g \in L^{q}[0,1]$, where $p>1, q>1$, with $\frac{1}{p}+\frac{1}{q}=1$. Then $h g \in L^{1}[0,1]$ and $\|h g\|_{1} \leq\|h\|_{p}\|g\|_{q}$. Further, if $h \in L^{1}[0,1]$ and $g \in L^{\infty}[0,1]$. Then $h g \in L^{1}[0,1]$ and $\|h g\|_{1} \leq\|h\|_{1}\|g\|_{\infty}$.

We establish the denumerably many positive solutions for the following three possible case for $\omega \in L^{p}[0,1]: 0<p<1, p=1, p=\infty$.

Theorem 3.3. Suppose $\left(H_{1}\right)$ and $\left(H_{2}\right)$ hold, let $\left\{\varrho_{k}\right\}_{k=1}^{\infty}$ be a sequence with $t_{k+1}<\varrho_{k}<t_{k}$. Let $\left\{E_{k}\right\}_{k=1}^{\infty}$ and $\left\{O_{k}\right\}_{k=1}^{\infty}$ be such that

$$
E_{k+1}<\varrho_{k}^{\eta-1} O_{k}<\beta O_{k}<E_{k}, k \in \mathbb{N},
$$


where

$$
\beta=\max \left\{\frac{1}{\varrho_{1}^{\eta-1} \sigma\left(\varrho_{1}\right) \int_{\varrho_{1}}^{1-\varrho_{1}} \chi(1, s) \psi(s) d s}, 1\right\}, \sigma\left(\varrho_{1}\right)=\max _{t \in[0,1]}\left\{1+\int_{\varrho_{1}}^{1-\varrho_{1}} \mathcal{H}(t, \tau) d \tau\right\} .
$$

Assume that

$$
\lambda=\max _{t \in[0,1]}\left\{\int_{0}^{1} \mathcal{H}(t, \tau) d \tau\right\}<\infty
$$

and $f$ satisfies

(A1) $f(\omega(t)) \leq \phi\left(K_{1} E_{k}\right)$ for all $t \in[0,1], 0 \leq \omega \leq E_{k}$, where $K_{1}<\frac{1}{(1+\lambda)\|\chi(1, s)\|_{q}\|\psi\|_{p}}$,

(A2) $f(\omega(t)) \geq \phi\left(\beta O_{k}\right)$ for all $t \in\left[\varrho_{k}, 1-\varrho_{k}\right], \varrho_{k}^{\eta-1} O_{k} \leq \omega \leq O_{k}$.

Then the bop (1.1) has denumerably many positive solutions $\left\{\omega_{k}\right\}_{k=1}^{\infty}$ such that $O_{k} \leq\left\|\omega_{k}\right\| \leq$ $E_{k}$ for $k=1,2,3 \cdots$.

Proof. Let $\Omega_{1, k}=\left\{\omega \in \mathcal{X}:\|\omega\|<E_{k}\right\}, \Omega_{2, k}=\left\{\omega \in \mathcal{X}:\|\omega\|<O_{k}\right\}$ be open subsets of $\mathcal{X}$. Let $\left\{\varrho_{k}\right\}_{k=1}^{\infty}$ be given in the hypothesis and we note that $t^{*}<t_{k+1}<\varrho_{k}<t_{k}<\frac{1}{2}$, for all $k \in \mathbb{N}$.

For each $k \in \mathbb{N}$, we define the cone $\mathcal{P}_{\varrho_{k}}$ by

$$
\mathcal{P}_{\varrho_{k}}=\left\{\omega \in \mathcal{X}: \omega(t) \geq 0, \min _{t \in\left[\varrho_{k}, 1-\varrho_{k}\right]} \omega(t) \geq \varrho_{k}^{\eta-1}\|\omega(t)\|\right\} .
$$

Let $\omega \in \mathcal{P}_{\varrho_{k}} \cap \partial \Omega_{1, k}$. Then, $\omega(s) \leq E_{k}=\|\omega\|$ for all $s \in[0,1]$. By $(A 1)$,

$$
\begin{aligned}
\|\mathcal{A} \omega\| & =\max _{t \in[0,1]}\left\{\int_{0}^{1} \chi(t, \tau) \phi^{-1}\left(\mathcal{I}_{0^{+}}^{\varepsilon}(f(\omega(\tau)))\right) d \tau\right. \\
& \left.+\int_{0}^{1} \mathcal{H}(t, \tau) \int_{0}^{1} \chi(\tau, s) \phi^{-1}\left(\mathcal{I}_{0^{+}}^{\varepsilon}(f(\omega(s)))\right) d s d \tau\right\} \\
& \leq \max _{t \in[0,1]}\left\{K_{1} E_{k} \int_{0}^{1} \chi(1, \tau) \psi(\tau) d \tau\right. \\
& \left.+K_{1} E_{k} \int_{0}^{1} \mathcal{H}(t, \tau) \int_{0}^{1} \chi(1, s) \psi(s) d s d \tau\right\} \\
& \leq K_{1} E_{k} \max _{t \in[0,1]}\left\{1+\int_{0}^{1} \mathcal{H}(t, \tau) d \tau\right\} \int_{0}^{1} \chi(1, \tau) \psi(\tau) d \tau \\
& \leq(1+\lambda) K_{1} E_{k} \int_{0}^{1} \chi(1, \tau) \psi(\tau) d \tau \\
& \leq(1+\lambda) K_{1} E_{k}\|\chi(1, \tau)\|_{q}\|\psi\|_{p} \\
& \leq E_{k} .
\end{aligned}
$$

Since $E_{k}=\|\omega\|$ for $\omega \in \mathcal{P}_{\varrho_{k}} \cap \partial \Omega_{1, k}$, we get

$$
\|\mathcal{A} \omega\| \leq\|\omega\| \text {. }
$$

Let $t \in\left[\varrho_{k}, 1-\varrho_{k}\right]$. Then,

$$
O_{k}=\|\omega\| \geq \omega(t) \geq \min _{t \in\left[\varrho_{k}, 1-\varrho_{k}\right]} \omega(t) \geq \varrho_{k}^{\eta-1}\|\omega\| \geq \varrho_{k}^{\eta-1} O_{k} .
$$


By $(A 2)$,

$$
\begin{aligned}
\|\mathcal{A} \omega\|= & \max _{t \in[0,1]}\left\{\int_{0}^{1} \chi(t, \tau) \phi^{-1}\left(\mathcal{I}_{0^{+}}^{\varepsilon}(f(\omega(\tau)))\right) d \tau\right. \\
& \left.+\int_{0}^{1} \mathcal{H}(t, \tau) \int_{0}^{1} \chi(\tau, x) \phi^{-1}\left(\mathcal{I}_{0^{+}}^{\varepsilon}(f(\omega(x)))\right) d x d \tau\right\} \\
\geq & \max _{t \in[0,1]}\left\{\int_{\varrho_{k}}^{1-\varrho_{k}} \chi(t, \tau) \phi^{-1}\left(I_{0^{+}}^{\varepsilon}(f(\omega(\tau)))\right) d \tau\right. \\
& \left.+\int_{\varrho_{k}}^{1-\varrho_{k}} \mathcal{H}(t, \tau) \int_{\varrho_{k}}^{1-\varrho_{k}} \chi(\tau, x) \phi^{-1}\left(\mathcal{I}_{0^{+}}^{\varepsilon}(f(\omega(x)))\right) d x d \tau\right\}
\end{aligned}
$$

That is,

$$
\begin{aligned}
\|\mathcal{A} z\| \geq & \max _{t \in[0,1]}\left\{\int_{\varrho_{1}}^{1-\varrho_{1}} \chi(t, \tau) \phi^{-1}\left(I_{0^{+}}^{\varepsilon}(f(\omega(\tau)))\right) d \tau\right. \\
& \left.+\int_{\varrho_{1}}^{1-\varrho_{1}} \mathcal{H}(t, \tau) \int_{\varrho_{1}}^{1-\varrho_{1}} \chi(\tau, x) \phi^{-1}\left(\mathcal{I}_{0^{+}}^{\varepsilon}(f(\omega(x)))\right) d x d \tau\right\} \\
\geq & \max _{t \in[0,1]}\left\{\beta O_{k} \varrho_{1}^{\eta-1} \int_{\varrho_{1}}^{1-\varrho_{1}} \chi(1, \tau) \psi(\tau)\right. \\
& \left.+\beta O_{k} \varrho_{1}^{\eta-1} \int_{\varrho_{1}}^{1-\varrho_{1}} \mathcal{H}(t, \tau) \int_{\varrho_{1}}^{1-\varrho_{1}} \chi(1, x) \psi(x) d x d \tau\right\} \\
\geq & \beta O_{k} \varrho_{1}^{\eta-1} \max _{t \in[0,1]}\left\{1+\int_{\varrho_{1}}^{1-\varrho_{1}} \mathcal{H}(t, \tau) d \tau\right\} \int_{\varrho_{1}}^{1-\varrho_{1}} \chi(1, x) \psi(x) d x \\
\geq & \beta O_{k} \varrho_{1}^{\eta-1} \sigma\left(\varrho_{1}\right) \int_{\varrho_{1}}^{1-\varrho_{1}} \chi(1, x) \psi(x) d x \\
\geq & O_{k}=\|\omega\| .
\end{aligned}
$$

Thus, if $\omega \in \mathcal{P}_{\varrho} \cap \partial \Omega_{2, k}$, then

$$
\|\mathcal{A} \omega\| \geq\|\omega\| .
$$

It is evident that $0 \in \Omega_{2, k} \subset \bar{\Omega}_{2, k} \subset \Omega_{1, k}$. From (3.6),(3.7), it follows from Theorem 3.1 that the operator $\mathcal{F}$ has a fixed point $\omega_{k} \in \mathcal{P}_{\varrho_{k}} \cap\left(\bar{\Omega}_{1, k} \backslash \Omega_{2, k}\right) \ni O_{k} \leq\left\|\omega_{k}\right\| \leq E_{k}$. The proof is completed.

For $p=1$, we have the following theorem.

Theorem 3.4. Suppose $\left(H_{1}\right)$ and $\left(H_{2}\right)$ hold, let $\left\{\varrho_{k}\right\}_{k=1}^{\infty}$ be a sequence with $t_{k+1}<\varrho_{k}<t_{k}$. Let $\left\{E_{k}\right\}_{k=1}^{\infty}$ and $\left\{O_{k}\right\}_{k=1}^{\infty}$ be such that

$$
E_{k+1}<\varrho_{k}^{\eta-1} O_{k}<\alpha O_{k}<E_{k}, k \in \mathbb{N},
$$

Assume that $f$ satisfies

(B1) $f(\omega(t)) \leq \phi\left(K_{2} E_{k}\right)$ for all $t \in[0,1], 0 \leq \omega \leq E_{k}$, where

$$
K_{2}<\min \left\{\frac{1}{(1+\lambda)\|\chi(1, \tau)\|_{\infty}\|\psi\|_{1}}, \beta\right\}
$$

and (A2). Then the bop (1.1) has denumerably many positive solutions $\left\{\omega_{k}\right\}_{k=1}^{\infty}$. Furthermore, $O_{k} \leq\left\|\omega_{k}\right\| \leq E_{k}$ for each $k \in \mathbb{N}$. 
Proof. For a fixed $k$, let $\Omega_{1, k}$ be as in the proof of Theorem 3.3 and let $\omega \in P_{\varrho_{k}} \cap \partial \Omega_{2, k}$. Again

$$
\omega(s) \leq E_{k}=\|\omega\|
$$

for all $s \in[0,1]$. By $(B 1)$ and Theorem 3.3,

$$
\begin{aligned}
\|\mathcal{A} \omega\|=\max _{t \in[0,1]}\left\{\int_{0}^{1} \chi(t, \tau) \phi^{-1}\left(\mathcal{I}_{0^{+}}^{\varepsilon}(f(\omega(\tau)))\right) d \tau\right. \\
\left.\quad+\int_{0}^{1} \mathcal{H}(t, \tau) \int_{0}^{1} \chi(\tau, x) \phi^{-1}\left(\mathcal{I}_{0^{+}}^{\varepsilon}(f(\omega(x)))\right) d x d \tau\right\} \\
\leq \max _{t \in[0,1]}\left\{K_{2} E_{k} \int_{0}^{1} \chi(1, \tau) \psi(\tau) d \tau\right. \\
\left.\quad+K_{2} E_{k} \int_{0}^{1} \mathcal{H}(t, \tau) \int_{0}^{1} \chi(1, x) \psi(x) d x d \tau\right\} \\
\leq K_{2} E_{k} \max _{t \in[0,1]}\left\{1+\int_{0}^{1} \chi(t, \tau) d \tau\right\} \int_{0}^{1} \chi(1, \tau) \psi(\tau) d \tau \\
\leq(1+\lambda) K_{2} E_{k} \int_{0}^{1} \chi(1, \tau) \psi(\tau) d \tau \\
\leq(1+\lambda) K_{2} E_{k}\|\chi(1, \tau)\|_{\infty}\|\psi\|_{1} \\
\leq E_{k} .
\end{aligned}
$$

Thus,

$$
\|\mathcal{A} \omega\| \leq\|\omega\|
$$

for $\omega \in \mathcal{P}_{\varrho_{k}} \cap \partial \Omega_{1, k}$. Now define $\Omega_{2, k}=\left\{\omega \in \mathcal{X}:\|\omega\|<O_{k}\right\}$. Let $\omega \in \mathcal{P}_{\varrho_{k}} \cap \partial \Omega_{2, k}$ and let $s \in\left[\varrho_{k}, 1-\varrho_{k}\right]$. Then, the argument leading to (3.7) can be done to the present case. Hence, the theorem.

Lastly, the case $p=\infty$.

Theorem 3.5. Assume that $\left(H_{1}\right)$ and $\left(H_{2}\right)$ hold. Let $\left\{E_{k}\right\}_{k=1}^{\infty}$ and $\left\{O_{k}\right\}_{k=1}^{\infty}$ be such that

$$
E_{k+1}<\varrho_{k}^{\eta-1} O_{k}<\beta O_{k}<E_{k}, k \in \mathbb{N},
$$

Assume that $f$ satisfies

(E1) $f(\omega(t)) \leq \phi\left(K_{3} E_{k}\right)$ for all $t \in[0,1], 0 \leq \omega \leq E_{k}$, where

$$
M_{3}<\min \left\{\frac{1}{(1+\lambda)\|\chi(1, \tau)\|_{1}\|\psi\|_{\infty}}, \beta\right\}
$$

and (A2). Then the bop (1.1) has denumerably many positive solutions $\left\{\omega_{k}\right\}_{k=1}^{\infty}$ such that $O_{k} \leq$ $\left\|\omega_{k}\right\| \leq E_{k}$ for $k=1,2,3, \cdots$. 
Proof. By $(E 1)$,

$$
\begin{aligned}
\|\mathcal{A} \omega\|=\max _{t \in[0,1]}\left\{\int_{0}^{1} \chi(t, \tau) \phi^{-1}\left(\mathcal{I}_{0^{+}}^{\varepsilon}(f(\omega(\tau)))\right) d \tau\right. \\
\left.\quad+\int_{0}^{1} \mathcal{H}(t, \tau) \int_{0}^{1} \chi(\tau, x) \phi^{-1}\left(\mathcal{I}_{0^{+}}^{\varepsilon}(f(\omega(x)))\right) d x d \tau\right\} \\
\leq \max _{t \in[0,1]}\left\{K_{2} E_{k} \int_{0}^{1} \chi(1, \tau) \psi(\tau) d \tau\right. \\
\left.\quad+K_{2} E_{k} \int_{0}^{1} \mathcal{H}(t, \tau) \int_{0}^{1} \chi(1, x) \psi(x) d x d \tau\right\} \\
\leq K_{2} E_{k} \max _{t \in[0,1]}\left\{1+\int_{0}^{1} \chi(t, \tau) d \tau\right\} \int_{0}^{1} \chi(1, \tau) \psi(\tau) d \tau \\
\leq(1+\lambda) K_{2} E_{k} \int_{0}^{1} \chi(1, \tau) \psi(\tau) d \tau \\
\leq(1+\lambda) K_{2} E_{k}\|\chi(1, \tau)\|_{1}\|\psi\|_{\infty} \\
\leq E_{k} .
\end{aligned}
$$

This shows that if $\omega \in \mathcal{P}_{\varrho_{k}} \cap \partial \Omega_{1, k}$, where $\Omega_{1, k}=\left\{\omega \in \mathcal{X}:\|\omega\|<E_{k}\right\}$, Then,

$$
\|\mathcal{A} \omega\| \leq\|\omega\|
$$

Define $\Omega_{2, k}=\left\{\omega \in \mathcal{X}:\|\omega\|<O_{k}\right\}$ and let $z \in \mathcal{P}_{\varrho_{k}} \cap \partial \Omega_{2, k}$. Then, the argument worked in the proof of Theorem 3.3 can be applied directly to get

$$
\|\mathcal{A} \omega\| \geq\|\omega\|
$$

This completes proof of the present theorem.

\section{EXAMPLES}

In this section, we present an example to check validity of our main results.

Example 4.1. Consider the following fractional order boundary value problem,

$$
\left.\begin{array}{c}
\mathfrak{D}_{0^{+}}^{3 / 4}\left(\phi\left(\mathfrak{D}_{0^{+}}^{5 / 2} \omega(t)\right)\right)+f(\omega(t))=0, t \in(0,1), \\
\omega(0)=\mathfrak{D}_{0^{+}}^{5 / 2} \omega(0)=0, \omega^{\prime}(0)=0, \mathfrak{D}_{0^{+}}^{3 / 4} \omega(1)=I_{0^{+}}^{2} \omega(1),
\end{array}\right\}
$$

where

$$
\phi(\omega)= \begin{cases}\frac{\omega^{3}}{1+\omega^{2}}, & \omega \leq 0 \\ \omega^{2}, & \omega>0\end{cases}
$$




$$
f(\omega)=\left\{\begin{array}{lc}
K_{1}^{2} E_{1}^{2}, & \omega>E_{1}, \\
\beta^{2} O_{k}^{2}+\frac{K_{1}^{2} E_{k}^{2}-\beta^{2} O_{k}^{2}}{E_{k}-O_{K}}\left(\omega-O_{k}\right), & O_{k} \leq \omega \leq E_{k}, k \in \mathbb{N}, \\
\beta^{2} O_{k}^{2}, & \tau_{k}^{\alpha-1} O_{k}<\omega<E_{k}, k \in \mathbb{N}, \\
K_{1}^{2} E_{k+1}^{2}+\frac{\beta^{2} O_{k}^{2}-K_{1}^{2} E_{k+1}^{2}}{\tau_{k}^{\alpha-1} O_{k}-E_{k+1}}\left(\omega-E_{k+1}\right), & E_{k+1}<\omega \leq \tau_{k}^{\alpha-1} O_{k}, k \in \mathbb{N}, \\
0, \quad & \omega=0, \\
t_{k}=\frac{31}{64}-\sum_{j=1}^{k} \frac{1}{4(j+1)^{4}}, k=1,2,3, \cdots
\end{array}\right.
$$

Let

$$
p=q=2, t_{k}=\frac{31}{64}-\sum_{j=1}^{k} \frac{1}{4(j+1)^{4}}, \text { for } k=1,2,3, \cdots, \varrho_{k}=\frac{1}{2}\left(t_{k}+t_{k+1}\right),
$$

then

$$
\varrho_{1}=\frac{15}{32}-\frac{1}{648}<\frac{15}{32}, \varrho_{k}>\frac{1}{5}
$$

and

$$
t_{k+1}<\varrho_{k}<t_{k}
$$

Therefore,

$$
\varrho_{k}^{\eta-1}>\frac{1}{5^{3 / 2}}, k=1,2,3, \cdots
$$

It is easy to see

$$
t_{1}=\frac{15}{32}<\frac{1}{2}, \text { for } k=1,2,3, \cdots, t_{k}-t_{k+1}=\frac{1}{4(k+2)^{4}} .
$$

Since $\sum_{k=1}^{\infty} \frac{1}{k^{2}}=\frac{\pi^{2}}{6}$ and $\sum_{k=1}^{\infty} \frac{1}{k^{4}}=\frac{\pi^{4}}{90}$, it follows that

$$
\begin{aligned}
t^{*}=\lim _{k \rightarrow \infty} t_{k} & =\frac{31}{64}-\sum_{j=1}^{\infty} \frac{1}{4(j+1)^{4}} \\
& =\frac{47}{64}-\frac{\pi^{4}}{360}>\frac{1}{5},
\end{aligned}
$$

$$
\begin{aligned}
\text { and }\|\psi\|_{2}= & {\left[\int_{0}^{1}\left[\phi^{-1}\left(\frac{s^{\varepsilon}}{\Gamma(\varepsilon+1)}\right)\right]^{2} d s\right]^{1 / 2}=\left[\int_{0}^{1}\left[\frac{s^{\varepsilon}}{\Gamma(\varepsilon+1)}\right]^{4} d s\right]^{1 / 2} \approx 0.5919429969 } \\
\lambda & =\max _{t \in[0,1]}\{\mathcal{H}(t, s)\} \\
& =\frac{\Gamma(\eta-\varepsilon) \Gamma(\eta+\delta)}{[\Gamma(\eta+\delta)-\Gamma(\eta-\varepsilon)] \Gamma(\eta) \Gamma(\delta)} \max _{t \in[0,1]}\left\{\int_{0}^{1} t^{\eta-1}(1-\tau)^{\delta-1} d \tau\right\} \\
& \approx 0.7506812224 \cdot \max _{t \in[0,1]}\left\{\frac{1}{2} t^{\eta-1}\right\} \\
& \approx 0.3753406112,
\end{aligned}
$$




$$
\begin{aligned}
\sigma\left(\varrho_{1}\right) & =1+\max _{t \in[0,1]}\left\{\int_{\varrho}^{1-\varrho_{1}} \mathcal{H}(t, \tau) d \tau\right\} \\
& =1+\frac{\Gamma(\eta-\varepsilon) \Gamma(\eta+\delta)}{[\Gamma(\eta+\delta)-\Gamma(\eta-\varepsilon)] \Gamma(\eta) \Gamma(\delta)} \max _{t \in[0,1]}\left\{\int_{\varrho}^{1-\varrho_{1}} t^{\eta-1}(1-\tau)^{\delta-1} d \tau\right\} \\
& =1.024617247
\end{aligned}
$$$$
\int_{0}^{1}[\chi(1, s)]^{2} d s=\frac{4}{65 \pi}, \text { it follows that }\|\chi(1, s)\|_{2}=\sqrt{\frac{4}{65 \pi}} \approx 0.1399582105
$$$$
\varrho_{1}^{\eta-1} \sigma\left(\varrho_{1}\right) \int_{\varrho_{1}}^{1-\varrho_{1}} \chi(1, s) \psi(s) d s=0.3193476457 \times 1.024617247
$$$$
\times \int_{\varrho_{1}}^{1-\varrho_{1}} \frac{(1-s)^{\eta-\varepsilon-1}-(1-s)^{\eta-1}}{\Gamma(\eta)} \frac{s^{\varepsilon}}{\Gamma(\varepsilon+1)} d s
$$$$
\leq 0.3193476457 \times 1.024617247 \times 0.08733438065
$$$$
=0.02857660458 \text {. }
$$

$$
\beta=\max \left\{\frac{1}{\varrho_{1}^{\eta-1} \sigma\left(\varrho_{1}\right) \int_{\varrho_{1}}^{1-\varrho_{1}} \chi(1, s) \psi(s) d s}, 1\right\}>34.99366054
$$

So, taking $\beta=35$. Thus,

$$
K_{1}<\frac{1}{(1+\lambda)\|\chi(1, s)\|_{q}\|\psi\|_{p}}=8.776300099 .
$$

So, taking, $K_{1}=8$ and Next, taking

$$
O_{k}=10^{-2(2 k+1)} \text { and } E_{k}=10^{-4 k},
$$

then

$$
\begin{aligned}
E_{k+1} & =10^{-(4 k+4)}<\frac{1}{5^{3 / 2}} \times 10^{-(4 k+2)}<\varrho_{k}^{\eta-1} O_{k} \\
& <O_{k}=10^{-(4 k+2)}<E_{k}=10^{-4 k}
\end{aligned}
$$

$\beta O_{k}=35 \times 10^{-2(2 k+1)}<8 \times 10^{-4 k}=K_{1} E_{k}$.

Also, $f$ satisfies conditions:

$$
\begin{aligned}
f(\omega) & \leq \phi\left(K_{1} E_{k}\right)=K_{1}^{2} E_{k}^{2}=64 \times 10^{-8 k}, \omega \in\left[0,10^{-4 k}\right] \\
f(\omega) & \geq \phi\left(\beta O_{k}\right)=\beta^{2} O_{k}^{2} \\
& =1225 \times 10^{-(8 k+4)}, \omega \in\left[\frac{1}{5^{3 / 2}} \times 10^{-2(2 k+1)}, 10^{-2(2 k+1)}\right] .
\end{aligned}
$$

Hence, by Theorem 3.2, the bvp (4.8) has denumerably infinitely many positive solutions $\left\{\omega^{[k]}\right\}_{k=1}^{\infty}$ with $10^{-6} \leq\left\|\omega^{[1]}\right\| \leq 10^{-4}, 10^{-10} \leq\left\|\omega^{[2]}\right\| \leq 10^{-8}, 10^{-14} \leq\left\|\omega^{[3]}\right\| \leq 10^{-12}$ and so on.

Acknowledgement. The authors thank to the referee for his appropriate comments and suggestions which help us to improve the manuscript. The author M. Rashmitha is thankful to DST-INSPIRE, Government of India, New Delhi for awarding JRF No. 2016/IF160480. 


\section{REFERENCES}

[1] Aghajani, A., Jalilian, Y. and Trujillo, J. J., On the existence of solutions of fractional integro-difierential equations, Fract. Calc. Appl. Anal., 15 (2012), No. 1, 44-69

[2] Bai, Z. and L, H., Positive solutions for a boundary value problem of nonlinear fractional differential equations, J. Math. Anal. Appl., 311 (2005), 495-505

[3] Benchohra, M., Graef, J. R. and Mostafai, F. Z., Weak solutions for nonlinear fractional difierential equations on reflexive Banach spaces, Electron. J. Qual. Theory. Diff. Eq., 54 (2010), 1-10

[4] Borisut, P., Kumam, P., Ahmed, I. and Sitthithakerngkiet, K., Nonlinear Caputo Fractional Derivative with Nonlocal Riemann-Liouville Fractional Integral Condition Via Fixed Point Theorems, Symmetry, 11 (2019), No. 6, 829

[5] Cabada, A. and G. Wang, G., Positive solutions of nonlinear fractional differential equations with integral boundary value conditions', J. Math. Anal. Appl., 389 (2012), 403-411

[6] Cadariu, L., Gavruţa, L. and Gavruça, P., Weighted space method for the stability of some nonlinear equations', Appl Anal Discrete Math., 6 (2012), No. 1, 126-139

[7] Delbosco, D. and Rodino, L., Existence and uniqueness for a nonlinear fractional differential equation, J. Math. Anal. Appl., 204 (1996) 609-625

[8] De Lemos, M. J., Turbulence in Porous Media: Modeling and Applications, Elsevier, 2012

[9] Ege, S. M. and Topal, F. S., Existence of positive solutions for fractional order boundary value problems, J. Applied Anal. Comp., 7 (2017), No. 2, 702-712

[10] Fen, F. T., Karaca, I. Y. and Ozen, O. B., Positive solutions of boundary value problems for p-Laplacian fractional differential equations, Filomat, 31 (2017), No. 5, 1265-1277

[11] Feng, M. Q., Zhang, X. M. and Ge, W. G., New existence results for higher-order nonlinear fractional differential equation with integral boundary conditions', Bound. Value Probl., Art. ID 720702, (2011), 20 pp.

[12] Gejji, V. D. and Babakhani, A., Analysis of a system of fractional differential equations, J. Math. Anal. Appl., 293 (2004), 511-522

[13] Guo, D. and Lakshmikantham, V., Nonlinear Problems in Abstract Cones', Academic Press, San Diego, 1988

[14] Kilbas, A. A., Srivastava, H. M. and Trujillo, J. J., Theory and applications of fractional differential equations, North-Holland Mathematics Studies, Elsevier Science B. V., Amsterdam., 204 (2006)

[15] Kilbas, A. A. and Trujillo, J. J., Differential equations of fractional order: Methods, results and problems II, Appl. Anal., 81 (2002), 435-493

[16] Lakshmikantham, V., Leela, S. and Vasundhara, J., Theory of Fractional Dynamic Systems, Cambridge Academic Publishers, Cambridge, 2009

[17] Leibenson, L. S., General problem of the movement of a compressible fluid in a porous medium', Izvestiia Akademii Nauk Kirgizskoi SSR., 9 (1983), 7-10.

[18] Li, K., Peng, J. and Gao, J., Nonlocal fractional semilinear Differential equations in separable Banach spaces', Electron. J. Differential Equations, 2013 (2013), No. 7, 1-7

[19] Liang, J., Liu, Z. and Wang, X., Solvability for a couple system of nonlinear fractional differential equations in a Banach space', Fract. Calc. Appl. Anal., 16 (2013), No. 1, 51-63

[20] Liu, X., Jia, M. and Ge, W., Multiple solutions of a p-Laplacian model involving a fractional derivative', Adv. Difference Equ., 2013 (2013), No. 1, 126

[21] Liu, X., Jia, M. and Xiang, X., On the solvability of a fractional differential equation model involving the p-Laplacian operator', Comput. Math. Appl., 64 (2013), No. 10, 3267-3275

[22] Liu, Z. H. and Lu, L., A class of BVPs for nonlinear fractional differential equations with p-Laplacian operator', Electron. J. Qual. Theory Differ. Equ., 70 (2012), 1-16

[23] Ljung, A. L. and Frishfelds, V., Lundström, T. S. and Marjavaara, B. D., Discrete and continuous modeling of heat and mass transport in drying of a bed of iron ore pellets', Drying Technol., 30 (2012), No. 7, 760-773

[24] Lu, H., Han, Z., Sun. S. and Liu, J., Existence on positive solutions for boundary value problems of nonlinear fractional differential equations with p-Laplacian', Adv. Differ. Equ., 2013 (2013), No. 1, 30

[25] Nakhushev, A. M., The Sturm-Liouville problem for a second order ordinary differential equation with fractional derivatives in the lower terms, Dokl. Akad. Nauk SSSR, 234 (1977), 308-311

[26] Podlubny, I., Fractional differential equations, Academic Press, San Diego, 1999

[27] Saadi, A. and Benbachir, M., Positive solutions for three-point nonlinear fractional boundary value problems', E. J. Qualitative Theory of Diff. Equ., 3 (2011), 1-19

[28] Sabatier, J., Agarwal, O. P. and Machado, J. A. T., Advances in fractional calculus: Theoretical developments and applications in physics and engineering', Springer, Dordrecht, 2007

[29] Tang, X. S., Yan, C. Y. and Liu, Q., Existence of solutions of two-point boundary value problems for fractional p-Laplace differential equations at resonance', J. Appl. Math. Comput., 41 (2013), No. 1, 119-131

[30] Tian, Y., Bai, Z. and Sujing, S., Positive solutions for a boundary value problem of fractional differential equation with $p$-Laplacian operator, Advances in difference equations, 2019 
[31] Torres, F. J., Existence of a positive solution for a boundary value problem of a nonlinear fractional differential equation, BIMS, 39 (2013), No. 2, 307-323

[32] Wang, L. and Zhai, C., Unique solutions for new fractional differential equations with $p$-Laplacian and infinite-point boundary conditions, Int. J. Dynamical Systems and Differential Equations, 9 (2019), No. 1, 1-13

[33] Wang, J. R., Zhou, Y. and Feckan, M., Abstract Cauchy problem for fractional differential equations', Nonlinear Dyn., 71:685, (2013), DOI 10.1007/s11071-012-0452-9

[34] Xu, X., Jiang, D., and Yuan, C., Multiple positive solutions for the boundary value problem of a nonlinear fractional differential equation, Nonlinear Analysis: Theory, Methods \& Applications, 71 (2009), No. 10, 4676-4688

[35] Yang, W., Positive solution for fractional q-difference boundary value problems with $p$-Laplacian operator, Bull. Malays. Math. Soc., 36 (2013), 1195-1203

[36] Zhang, S., Existence of positive solution for some class of nonlinear fractional differential equations, J. Math. Anal. Appl., 278 (2003), 136-148

[37] Zhang, S. Q., Existence results of positive solutions to fractional differential equation with integral boundary conditions', Math. Bohem., 135 (2010), No. 2, 299-317

Department of Applied Mathematics

COLLEGE OF SCIENCE AND TECHNOLOGY

ANDHRA UNIVERSITY, VISAKHAPATNAM

INDIA-530003

Email address: khuddush89@gmail.com 